Der Smart als Beispiel für neue Wege zur Öko-Effizienz im Verkehrsbereich

\title{
Schritt zum Mobilitätsmarketing
}

\begin{abstract}
Bislang haben umweltpolitische Akteure den Vertretern der Automobilwirtschaft häufig ein Dilemma unterstellt: Pläne für verbrauchsoptimierte Autos lägen seit langem in der Schublade, die Vermarkfung eines umweltgerechten Fahrzeuges sei nicht profitabel genug. Mittlerweile können die Automobilhersteller nicht nur widersprechen, sondern auch belegen: Sowohl MCC smart als auch andere Hersteller wagen den Schritt zum umweltorientierten Produkt.
\end{abstract}

$\mathbf{P}$ Von Reinbard Hoßfeld rodukte wie der smart entsprechen in ihren umweltrelevanten Eigenschaften dem vordersten Stand der Technik. Der smart selbst löst hinsichtlich Ökologie in der Produktion, Recycling-Rate und anderer Eigenschaften stille Revolutionen aus. Ökologische Aspekte werden zur Antriebsfeder eines gesunden Wettbewerbs. Umweltranking-Listen gewinnen an Bedeutung, und die Automobilhersteller versuchen mit ihren Mitteln, vordere Plätze einzunehmen.

Obwohl die Umweltthemen derzeit in der Öffentlichkeit nicht höchste Priorität haben, ist das umweltgerechte Fahrzeug doch anerkanntes Marketingziel. Diese Entwicklung ist zu begrüßen und wichtig. Wir wollen damit auch aus ökonomischen Gründen ein Produkt, das die umweltspezifischen Eigenschaften optimiert. Das zukunftsorientiert ist. Erfolg des Produktes bedeutet dann auch Erfolg für die Umwelt. Wer die Fahrzeugeigenschaften unter Umweltgesichtspunkten optimiert, erzielt das beste Ergebnis.

Wer die Fahrzeugeigenschaften unter Umweltgesichtspunkten optimiert, erzielt bereits das beste Ergebnis? Stop. Hier wissen wir auch als Automobilbauer heute mehr.

\section{Neve Wege der Mobilität}

Auf breiter Basis akzeptiert scheint heute folgende Aussage: Öko-Effizienz beruht auch darauf, technische Entwicklungen durch konzeptionelle Neuwege abzustützen. Quantensprünge sind nur durch neue Ansätze zu realisieren.

Mit dem smartmove Mobilitätskonzept geht der smart einen solchen Neuweg. Erstmalig vermarktet ein Automobilhersteller nicht nur die Mobilitätsleistung des Kernproduktes Auto, sondern ergänzt dieses Kernangebot durch Angebote aus dem Bereich des öffentlichen Verkehrs. Der smart Kunde in Deutschland kann zum Beispiel die Fahrzeuge der meisten deutschen Car Sharing-Anbieter nutzen, oder kann sich für seine Ankunft mit dem ICE Sprinter der DB einen smart als Anschlußmobilität buchen. Stadttouristen und Nutzer des öffentlichen Verkehrs können in Städten wie Zürich, Stuttgart und Karlsruhe einen smart als verlängerten Arm der Kommune bzw. des lokalen Verkehrsbetriebes nutzen. Und weil dies nur Erfolg hat, wenn es dem Fahrer Spaß und sichtbare Vorteile bringt, können die Fahrer der öffentlichen (und auch der im Privatbesitz befindlichen) smart in Deutschland-weit mehr als 50 Parkhäusern spezielle Parkplätze, teilweise sogar zu reduzierten Tarifen nutzen. Weitere Projekte, auch nach dem Beispiel der von smart in der Schweiz angebotenen smartmove TravelCard (verbilligte Reisen mit der Schweizer Bundesbahn, Zugriff auf mehr als 1000 CarSharing Fahrzeuge) werden derzeit in Deutschland und der Schweiz realisiert und ab Mitte 1999 auch für die übrigen smart Märkte vorbereitet.

Diese Auswahl an Mobilitätsprodukten zeigt, daß sich smart hier auf Neuland begibt. Neu ist, daß sich ein Automobilhersteller für die Nutzung des öffentlichen Verkehrs einsetzt. Daß er seinen Kunden die Nutzung der CarSharing-Angebote möglich macht. Daß er für den Fall der Fälle seinen Kunden auch in die Lage versetzt, auf andere Fahrzeuge zuzugreifen.

Selbstverständlich läßt sich dies bei smart gerade deshalb realisieren, weil das Kernprodukt smart die Basis für smartmove Mobilitätsangebote schafft: Wer ein für den Stadtverkehr spezialisiertes zweisitziges Fahrzeug anbietet, dem helfen solche Mobilitätsangebote auch bei der Vermarktung des Kernproduktes. Mancher wird sagen, das smart City Coupé braucht diese Angebote für einen Teil der Kundensegmente auch. Dem möchte ich nicht widersprechen. Denn um genau diese Kundensegmente, die empfänglich für intermodale Nutzungskonzepte und ähnliches sind, geht es uns doch bei der Optimierung ökologischer Mobilitätsformen.

Für die ökologisch Interessierten macht deshalb auch die umgekehrte Sichtweise Sinn: Wer intermodale Mobilität unterstïtzen möchte, der muß auch beim Auto darauf achten, die richtige Basis zu schaffen. Der muß zu einem Fahrzeug greifen, das Raum läßt für die spezifischen Vorteile anderer Verkehrsträger. Der braucht ein Auto, das die richtigen Dinge richtig tut. Aber eben nur die.

MCC smart bietet ein Fahrzeug für den innerstädtischen motorisierten Individualverkehr an. Und wir arbeiten an der bedarfsorientierten Bereitstellung anderer Fahrzeuge und anderer Verkehrsträger. Nach sechs Monaten Laufzeit der ersten Pilotrealisierungen liegen gute Erfahrungen hinsichtlich der Nutzung durch die Kunden vor. Dies gibt uns die Sicherheit, das smartmove Mobilitätskonzept weiter auszubauen. Gleichzeitig wird, die Attraktivität des Kernproduktes smart City Coupé weiter gesteigert.

Denn spätestens hier holt uns eine andere Facette der Öko-Effizienz ein: Das smartmove Mobilitätskonzept kann nur erfolgreich sein, wenn auch das Kernprodukt smart City Coupé erfolgreich ist. Und dafür muß der smart wie alle anderen Fahrzeuge auch durch die Fahrzeugeigenschaften überzeugen können. Je erfolgreicher der smart, desto mehr Potentiale werden frei für ökologische Belange.

Wir formulieren gerne überspitzt: Wer nicht einen smart, sondern ein anderes Fahrzeug kauft, wird weniger wahrscheinlich zum intermodalen Verkehrsnutzer. Und trägt deshalb dazu bei, daß Verkehrsträger nicht für ihre jeweiligen Optimaleinsatzgebiete genutzt werden.

Wir werden den Beitrag des smart zur Ökologie nicht nur dadurch belegen können, daß mit dem smart weniger Treibstoff und weniger Herstellungsenergie verbraucht wird, daß eine hohe Recycling-Rate erzielt wurde. Sondern auch dadurch, daß ein beträchtlicher Anteil an Personenkilometern auf andere Verkehrsträger verlagert wird und daß smart Kunden nur im tatsächlichen Bedarfsfall sich für die Nutzung eines größeren Fahrzeuges entscheiden.

\section{Der Autor}

Reinhard HoBfeld ist Leiter Mobility Systems der MCC smart GmbH.

Kontakt: Industriestr. 8D, 71272 Renningen.

Tel. 07031/ 90-4750, Fax -4999. 
(c) 20I0 Authors; licensee IÖW and oekom verlag. This is an article distributed under the terms of the Creative Commons Attribution Non-Commercial No Derivates License (http://creativecommons.org/licenses/by-nc-nd/3.o/), which permits unrestricted use, distribution, and reproduction in any medium, provided the original work is properly cited. 